\title{
A Simplified Multipath Component Modeling Approach for High-Speed Train Channel Based on Ray Tracing
}

\author{
Jingya Yang, ${ }^{1}$ Bo Ai, ${ }^{1}$ Danping He, ${ }^{1}$ Longhe Wang, ${ }^{1} Z_{\text {hangdui Zhong, }}{ }^{1}$ and Andrej Hrovat ${ }^{2}$ \\ ${ }^{1}$ State Key Laboratory of Rail Traffic Control and Safety, Beijing Jiaotong University and Beijing Engineering Research Center of \\ High-Speed Railway Broadband Mobile Communications, Beijing 100044, China \\ ${ }^{2}$ Department of Communication System, Jožef Stefan Institute, SI-1000 Ljubljana, Slovenia \\ Correspondence should be addressed to Bo Ai; boai@bjtu.edu.cn
}

Received 17 May 2017; Accepted 30 August 2017; Published 19 October 2017

Academic Editor: Gonzalo Vazquez-Vilar

Copyright (c) 2017 Jingya Yang et al. This is an open access article distributed under the Creative Commons Attribution License, which permits unrestricted use, distribution, and reproduction in any medium, provided the original work is properly cited.

\begin{abstract}
High-speed train (HST) communications at millimeter-wave (mmWave) band have received a lot of attention due to their numerous high-data-rate applications enabling smart rail mobility. Accurate and effective channel models are always critical to the HST system design, assessment, and optimization. A distinctive feature of the mmWave HST channel is that it is rapidly time-varying. To depict this feature, a geometry-based multipath model is established for the dominant multipath behavior in delay and Doppler domains. Because of insufficient mmWave HST channel measurement with high mobility, the model is developed by a measurementvalidated ray tracing (RT) simulator. Different from conventional models, the temporal evolution of dominant multipath behavior is characterized by its geometry factor that represents the geometrical relationship of the dominant multipath component (MPC) to HST environment. Actually, during each dominant multipath lifetime, its geometry factor is fixed. To statistically model the geometry factor and its lifetime, the dominant MPCs are extracted within each local wide-sense stationary (WSS) region and are tracked over different WSS regions to identify its "birth" and "death" regions. Then, complex attenuation of dominant MPC is jointly modeled by its delay and Doppler shift both which are derived from its geometry factor. Finally, the model implementation is verified by comparison between RT simulated and modeled delay and Doppler spreads.
\end{abstract}

\section{Introduction}

Characterized by high mobility, the high-speed train (HST) scenario is expected to be a typical scenario in the fifthgeneration (5G) wireless communication systems [1]. To enable intelligent transportation system, various high-datarate services are required in this scenario [2-4], for example, high definition (HD) video surveillance, and onboard realtime connectivity. For satisfying these services, applying advanced technologies in HST communications, including millimeter-wave (mmWave), mobile relay station (MRS), and smart antennas, has been studied in the literature [58]. Beforehand, to design these advanced technologies and evaluate their performance, accurate channel models are necessary. However, existing channel models at mmWave band mostly focus on indoor and static scenarios [9-13], without considering the rapidly time-varying HST channels between transmitter $(T x)$ and MRS in open space scenario.
Due to these facts, mmWave HST channel modeling has been underresearched in the literatures.

For modeling approaches, the current HST channel models can be roughly classified into three categories [5]: deterministic, stochastic, and geometry-based stochastic modeling.

Deterministic modeling is mainly based on geometrical optics or, more generally, on solving Maxwell's equations. Scenario reconstruction is the first and key step, which gives detailed geometries, specific locations of $T x$ and receiver $(R x)$, and so on. Then, combining with antenna configuration the complex attenuation, delay, angle, and polarization information of the propagation waves are obtained through intensive electromagnetic simulation. Therefore, the deterministic models are approximately exact. For HST channels deterministic modeling is proposed in [14-18]. In [14], the temporal evolutions of delay spread and Doppler spread at $5.2 \mathrm{GHz}$ in orthogonal frequency division multiplexing 
(OFDM) HST communication system are evaluated based on ray tracing $(\mathrm{RT})$. Similarly, $[15,16]$ using RT analyze HST channel characteristics, for example, path loss (PL), delay spread (DS), and angle spread (AS), at $930 \mathrm{MHz}$ and $5.25 \mathrm{GHz}$, respectively. As for these studies, time-varying characteristics in HST channels are analyzed in the nonmmWave band. Moreover, in [17] a linear HST environment is conducted and RT simulation at $60 \mathrm{GHz}$ for analysis of DS and AS changing over time is performed. In [18], adopting RT at $60 \mathrm{GHz}$ as well, six HST scenario modules are modeled and the DS for different modules are compared. However, in $[17,18]$ the validation by measurement in the HST deterministic models at mmWave band, which is crucial in channel modeling, is not performed. Furthermore, such modeling approach represented by RT fails in the situation as it is too complicated to reconstruct the exact scenario. In addition, the extensive computational resources are needed, which is not suitable for communication link-level and system-level simulations. Thus, stochastic modeling which is void of exact geometrical assumption and is computationally less demanding has been proposed.

Stochastic modeling provides the statistical manner and conditional dependencies between different channel parameters. Although such modeling approach gives very little insight in an instantaneous channel structure, it is useful in presenting the general characteristic of a typical scenario [19]. From this point of view the stochastic modeling is practically useful. The existing stochastic models for HST channels mainly focus on the narrowband [20-24]. For example, at $930 \mathrm{MHz}$, the PL, shadow fading, Rician $K$-factor, and decorrelation distance are stochastically modeled based on various HST measurements [20-23]. Their time-varying characteristics are described by Markov-chains [24]. Moreover, for wideband HST channels, the rough position-based tapped delay line channel models with specified Doppler shifts for open space scenario and hilly scenario are proposed in [25, 26], respectively. Nevertheless, comparing with deterministic modeling the stochastic models cannot keep the spatial consistency which is crucial in time-varying HST channel modeling [27]. Furthermore, the nonstationarity caused by high mobility of MRS is not characterized in these HST stochastic models.

To address the nonstationarity in time-varying channel, Bello proposed the wide-sense stationary (WSS) concept [28]. Under the WSS assumption the channel can be stationary over a limited time region and therein the stochastic modeling becomes physically meaningful. Particularly, the power delay profile (PDP) in delay domain and Doppler power spectral density (DSD) in Doppler domain completely describe the second-order statistics of time-varying channels $[29,30]$.

The third modeling approach, the geometry-based stochastic channel model (GSCM), is useful in nonstationary channel [31]. This is the type of modeling approach we aim for in this paper. Within the GSCMs specific locations of $T x, R x$, and scatterers are incorporated, where the distribution for scatterers is predefined by the statistical law. The standard channel models, like rural macrocell (RMa) scenario in the WINNER II channel model [32] and moving networks scenario in the IMT-A channel model [33], have considered the HST scenario. However, they are for the non-mmWave band. In [34], the narrowband GSCM is modeled and the scattering components are represented by the measurement-defining cluster. In [35], the regular shaped GSCM is developed by considering time-varying model parameters, that is, angle of departure (AoD) and angle of arrival (AoA). It provides the insights into the relationship of channel characteristics and certain geometry. However, due to oversimplified propagation environment the parametrization work from measurements would be hard [36]. In [37] the GSCM with random-cluster is proposed in delay and Doppler domains at $2.14 \mathrm{GHz}$. And yet for the limited bandwidth, multipath components (MPCs) are unresolvable and only a LoS cluster is modeled according to LoS geometry. While these GSCMs might not be scalable to the mmWave HST channels, they provide some geometrical insights for the HST channel modeling.

In this paper, we propose a geometry-based dominant multipath model for the mmWave HST channel. It fully depicts the temporal evolution of dominant multipath in the delay and Doppler domains. The modeling approach is simple and effective. Without using intensive RT or numerical simulations, the proposed modeling approach is based on the geometry factor extracted from the propagation environment. Through the right triangle constructed by the geometry factor, $T x$ location, and instantaneous location of MRS, the time-varying delay and Doppler shift can be derived. The amplitude of dominant multipath is modeled by the delay and Doppler shift which is presented first. The amplitude modeling implies the correlation between the delay and angle domains in the mmWave HST channel.

The proposed modeling approach shows insight into the relationship between the delay and Doppler shift for dominant MPCs in the mmWave HST channels. A similar modeling approach is introduced in $[19,38]$, where only single bounced rays were included. The proposed approach is capable of modeling first-order reflected paths (FRPs) and second-order reflected paths (SRPs), and the reflected paths strictly obey specular reflection law. Moreover, the number of newly dominant paths, their lifetimes, geometry factors (for the delay and Doppler shift calculation), and complex attenuation are statistically modeled and parameterized. A detailed implementation of the proposed model is presented, which is verified by comparison with measurement-validated RT. This simple and effective modeling approach is practical for system-level simulation and communication network evaluation.

The remainder of this paper is structured as follows. In Section 2, the RT simulator is validated through the measured data obtained in the mmWave HST channel and then the temporal evolution of PDP and DSD simulated by the validated RT in the mmWave HST scenario is discussed. Section 3 describes the structure of the proposed model, and the dominant paths are detected and tracked using geometry factors. Section 4 shows the model parameters. Section 5 presents the model implementation and validation. This paper concludes in Section 6. 


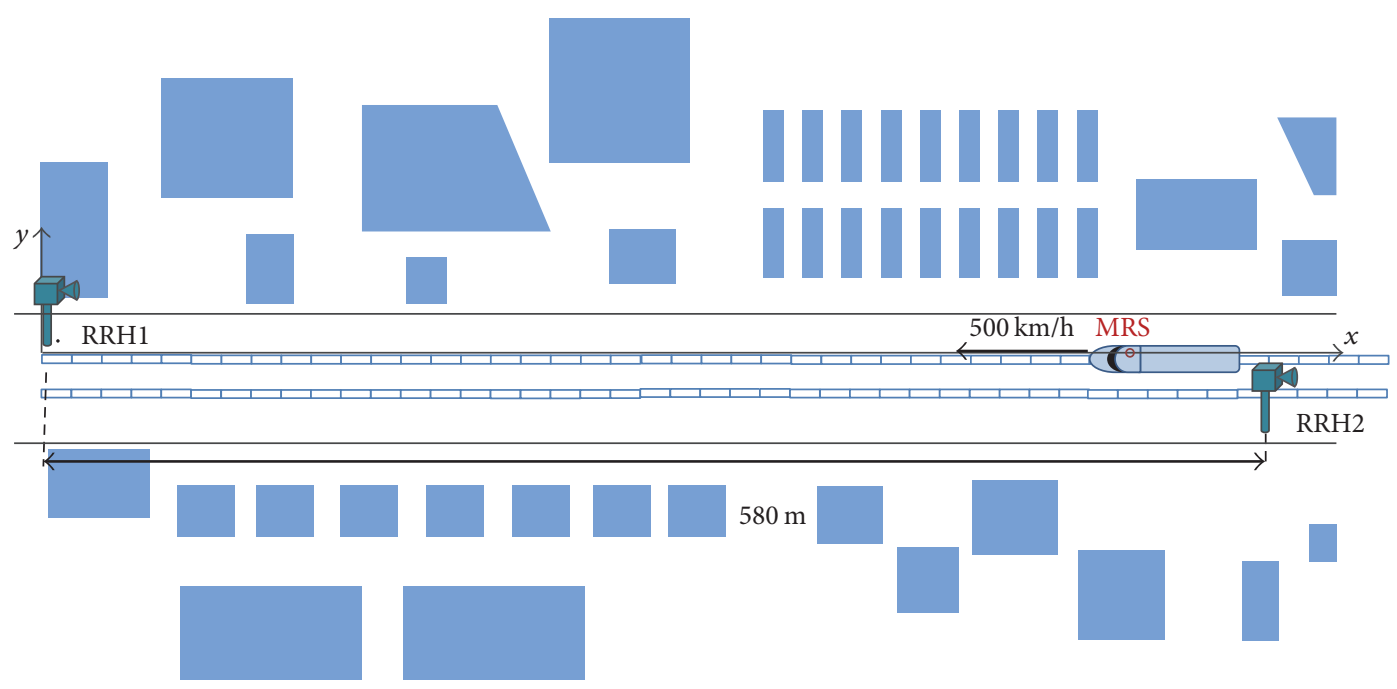

Figure 1: Top view of mmWave HST scenario deploying the MRS.

\section{Ray Tracing Validation and Simulation}

The typical mmWave HST scenario is shown is Figure 1, whose construct is proposed in the 3rd Generation Partnership Project (3GPP) [6]. The remote radio head (RRH) is placed $5 \mathrm{~m}$ from the track. The MRS is deployed on the top of the train. In addition, with the longitudinal separation of $580 \mathrm{~m}$ three RRHs are organized as a group connected to the baseband processing unit (BBU), which is a new cellular architecture for the mmWave HST communication system.

Due to deficient mmWave HST channel measurement with high mobility, development of the modeling approach is based on RT simulator. Therefore, first we will validate the RT simulator by the channel measurement which is performed in the mmWave HST scenario. In detail, the measurement campaign conducted in Japan was performed at $93.2 \mathrm{GHz}$ and with $2 \mathrm{GHz}$ bandwidth [39]. The frequency band was chosen in this measurement for two primary reasons. One is to consider the coexistence with active and passive services in the HST scenarios [40], and the other is for the high data transmission rate. The authors in [41] demonstrate that the attenuation of the $90 \mathrm{GHz}$ band millimeter-wave in the atmosphere is small compared with millimeter-waves of the other frequency bands. The MRS moved along the track and the RRH-MRS distance ranged from $20 \mathrm{~m}$ to $350 \mathrm{~m}$. Tx and $R x$ antennas were installed on RRH and MRS with the same half power beam width (HPBW), respectively. The antenna settings are summarized in Table 1. Note in RT simulation the antenna pattern is inserted by the Jones vector at all the angles of arrival or departure. These Jones vectors cover the gain and the polarization of the antennas. The same operation was done and reported in [15]. Moreover, the front view of typical structure in the measured scenario is shown in Figure 2.

2.1. Ray Tracing Validation. RT simulation was executed in the measured scenario with settings listed in Table 1. Specifically, the LoS path, reflected paths (up to the third order), and scattering rays are simulated. Since there is no

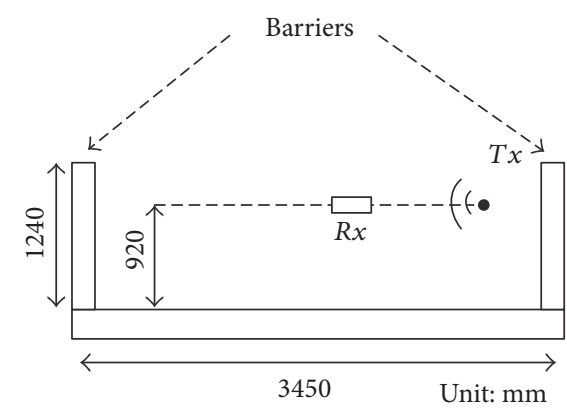

FIGURE 2: Front view of typical structure in the measured mmWave HST scenario.

TABLE 1: 93.2 GHz HST channel measurement settings.

\begin{tabular}{lc}
\hline Parameter & Setting \\
\hline$T x-R x$ antenna types & Directional horn antenna \\
$T x-R x$ antenna gains & $25 \mathrm{dBi}$ \\
$T x-R x$ antenna HPBWs & $10^{\circ} / 10^{\circ}(\mathrm{Az} . / \mathrm{El})$. \\
$T x-R x$ antenna heights & $0.92 \mathrm{~m}$ \\
$T x$ output power & $-5 \mathrm{dBm}$ \\
Center frequency & $93.2 \mathrm{GHz}$ \\
Bandwidth & $2 \mathrm{GHz}$
\end{tabular}

obstacle between $T x$ and $R x$, the diffraction mechanism is not included in the RT simulation. Since the mmWave HST channel was not measured dynamically, the measured channel characteristic in Doppler domain cannot be obtained. In addition, only the received power has been presented in [39] with no information about the delay domain. Due to the limitations for the measurement campaign, just the large-scale and small-scale parameters between measured and simulated channel are compared for validation. 


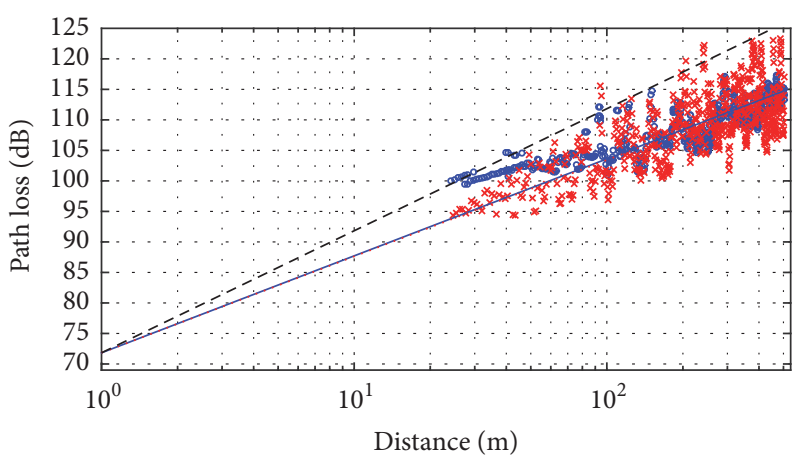

- Measured

$\times$ Simulated

$-n_{\text {meas }}=1.6, \sigma_{\text {meas }}=2.7 \mathrm{~dB}$

… $n_{\text {simu }}=1.6, \sigma_{\text {simu }}=2.0 \mathrm{~dB}$

- - - Free space, $n=2$

FIgURE 3: Directional path loss models estimated by measured and RT simulated channels, respectively, and compared to the free space propagation model.

We separate large-scale fading and small-scale fading by a sliding/overlapped window (size of 20 wavelengths) with an interval of ten wavelengths $[25,42]$.

2.1.1. Large-Scale Fading Validation. The decibel-valued path loss (PL) model is given by a linear Least Squares (LS) regression of the large-scale fading

$$
\mathrm{PL}[\mathrm{dB}](d)=\mathrm{PL}\left(d_{0}\right)+10 \bar{n} \log _{10}\left(\frac{d}{d_{0}}\right)+X_{\sigma},
$$

where $d$ is the distance between the Tx and $R x$ in meters and $\operatorname{PL}\left(d_{0}\right)$ is the frequency-dependent free space path loss at reference distance $d_{0}$, generally $d_{0}=1 . \bar{n}$ refers to the average path loss exponents (PLE) over the observed distance. Moreover, for modeling the shadow fading, $X_{\sigma}$ denotes the zero-mean lognormal random value with standard deviation $\sigma$, that is, shadow factor (SF). If $d_{0}$ and frequency are known, $\mathrm{PL}\left(d_{0}\right)$ in (1) is fixed. Hence, for large-scale fading validation, we compare the two parameters, that is, PLE and SF.

The measured and simulated large-scale fading are shown in Figure 3, where their respective PLE and SF are given and the free space path loss at $93.2 \mathrm{GHz}$ is added for comparison. The same PLE, that is, 1.6 , is estimated from the two channels. The SF for the measured channel is $2.7 \mathrm{~dB}$, which is just slightly higher than the simulated SF of $2.0 \mathrm{~dB}$. The good match of the PLE and SF indicates reasonable agreement in large-scale fading. In addition, due to the superposition of MPCs, the PLE in the HST scenario is lower than the PLE from free space.

2.1.2. Small-Scale Fading Validation. The empirical cumulative distribution functions (CDFs) of measured and RT simulated amplitudes for small-scale fading are shown in Figure 4. There is a good match between measured and RT simulated statistical properties in small-scale fading. Moreover, the mean vales of the measured and RT simulated amplitudes are just slightly different; that is, $\mu_{\text {meas }}=0.98$ and

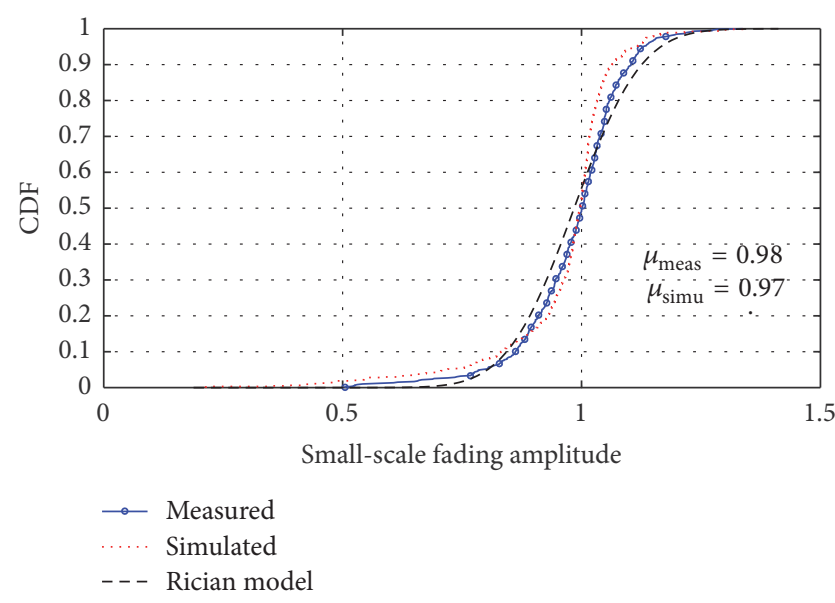

FIGURE 4: Comparison of CDFs between measured and simulated amplitude of small-scale fading. The Rician distribution is given for modeling.

$\mu_{\text {simu }}=0.97$. Due to existent LoS path, the Rician distribution is adopted to fit the distribution. Figure 4 shows that the Rician distribution can accurately characterize the amplitude of small-scale fading. The Rician fitted curve is derived from the measurement data.

The validation confirms that the RT simulator is able to accurately reproduce mmWave channel characteristics in HST scenario. It is worth noting that this scenario is characterized by its effective reflectors, most of which are trackside walls. Actually, the trackside walls are not necessarily from the barriers next to the track. Assuming that in the scenario the main effective reflectors are perpendicular to the ground, such as buildings and tunnel walls, the RT simulator should work adequately. Due to the measurement-validated RT we have the opportunity to extend the limited HST measurement scenario to a general mmWave HST scenario.

2.2. Ray Tracing Simulation. The simulated scenario is a typical mmWave HST environment shown in Figure 1. For the general RT simulation, the antenna heights and patterns in measured scenario have been changed and different antenna heights are set, that is, $3.2 \mathrm{~m}$ at $T x$ and $3 \mathrm{~m}$ at $R x$. The antenna heights for the RT simulation are raised compared with the measurement settings and exceed the heights of the trackside barriers as shown in Figure 2. Specifically, to raise the antenna heights is aimed at breaking the semiclosed space constructed by trackside barriers. Then, the impact of trackside buildings (blue blocks in Figure 1) can be increased, just like in the general propagation environment. To characterize the propagation channel in the mmWave HST scenario, the omnidirectional, vertically polarized pattern is used at $T x$ and $R x$, instead of the directional patterns. The movement range of MRS is from RRH2 to RRH1 with length of $580 \mathrm{~m}$, shown in Figure 1. The MRS speed is set to $500 \mathrm{~km} / \mathrm{h}$ that is the top speed discussed in 3GPP [6]. In addition, the parameter setting for the RT simulator is the same as above.

In this paper, we focus on the channel characteristics in delay and Doppler domains which are crucial in frame structure design (time domain) [43] and subcarrier spacing 


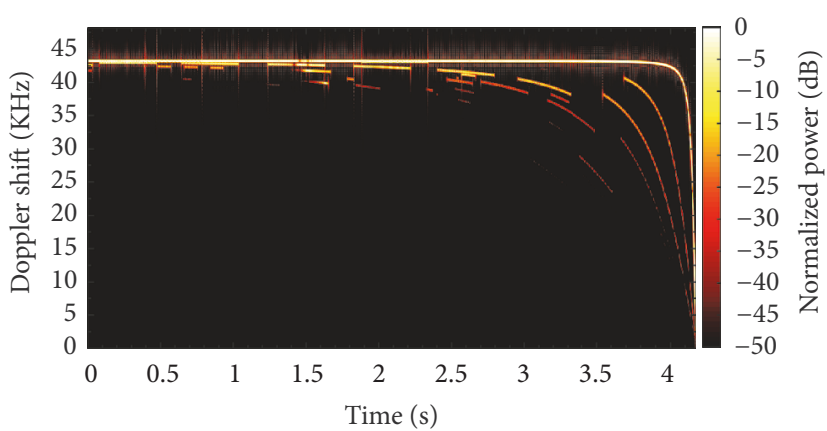

(a) Doppler power spectral density (DSD)

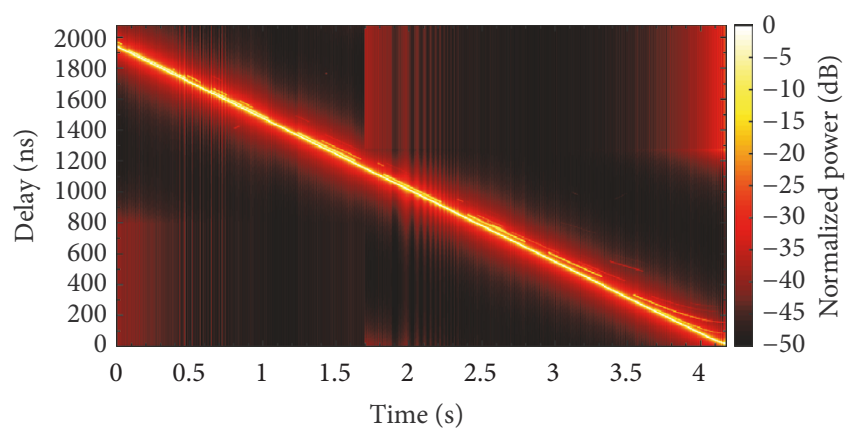

(b) Power delay profile (PDP)

FIgURE 5: The temporal evolution of power spectral density in Doppler and delay domains for the mmWave HST channel, respectively.

selection (frequency domain) [44]. Particularly for Doppler analysis, the sampling interval should be less than halfwavelength, that is, $1.6 \mathrm{~mm}$ (carrier frequency is $93.2 \mathrm{GHz}$ ), which indicates the huge data volume for RT simulation. During the MRS movement 720747 snapshots are simulated with a snapshot repetition time of $5.79 \mu \mathrm{s}$. Note that, for reducing the volume of simulated data, only the IRs in time and frequency domains for each snapshot are stored and the multipath property, that is, rays absolute delay, angle, electrical field, and so on, has not been exported from RT. The simulated data are stored in the same format as the data recorded in the channel measurement campaign, and then needed channel characteristics are extracted from the IRs, which makes the proposed approach general.

Analyses of the RT simulated data showed that the observed fading process in mmWave HST channel is nonstationary. Due to the propagation environment changing with a finite rate, the nonstationarity can be overcome by consecutive local WSS regions [45], that is, WSS time windows $\Delta W$. The local scattering function (LSF) introduced in [46] is used to determine the size of $\Delta W$. To ensure the stationarity analysis throughout, the minimum stationary time interval $3.6 \mathrm{~ms}$ is chosen for $\Delta W$. Therefore, within each WSS time window the statistics of delay and Doppler domains are locally stationary and we can average the local PDP and DSD within each WSS time window as introduced in [47].

For the mmWave HST channel the temporal evolutions of DSD and PDP are shown in Figure 5. Within each WSS time window, we normalize the instantaneous DSD and PDP to their own maximum values and set the cutoff threshold of $50 \mathrm{~dB}$ below the strongest peak. The continuous Doppler and delay trajectories of the time-varying LoS path can be easily distinguished in Figures 5(a) and 5(b), respectively. Obviously, the LoS path always exists. The other discontinuous Doppler trajectories in Figure 5(a) present at certain consecutive WSS time windows and then vanish. Their corresponding delay trajectories are shown in Figure 5(b). Since the continuous or discontinuous trajectories seem to follow certain curves, the underlying geometry for them will be found hereafter. Moreover, it is obvious that the multipath resolution is higher in Doppler domain than in delay domain. Thus, for clarity, the following illustrations are mostly in Doppler domain.

\section{Geometry-Based Dominant Multipath Channel Model and Data Processing}

3.1. Geometry-Based Dominant Multipath Channel Model. The channel model is based on the channel spreading function [37]. At time $t_{i}$, the channel spreading function is expressed as the superposition of $N\left(t_{i}\right)$ paths

$$
h\left(t_{i} ; \tau, v\right)=\sum_{l=1}^{N\left(t_{i}\right)} a_{l}\left(t_{i}\right) \cdot \delta\left(\tau-\tau_{l}\left(t_{i}\right)\right) \cdot \delta\left(\nu-v_{l}\left(t_{i}\right)\right)
$$

where $\delta(\cdot)$ is the Dirac delta function and $a_{l}\left(t_{i}\right), \tau_{l}\left(t_{i}\right)$, and $v_{l}\left(t_{i}\right)$ represent the complex attenuation, delay, and Doppler shift of the $l$ th path observed at time $t_{i}$, respectively. For the HST channels, the behavior of each path, that is, complex attenuation, delay, and Doppler shift, changes over time. Particularly, due to the high-speed MRS, the MPCs, except LoS path, frequently appear in the "birth" and "death" process, shown in Figure 5. For convenience, we define three sets of the path in (2) as follows [45]:

(i) $\mathscr{L}_{i \rightarrow i}$ is the set of dominant paths that are firstly detected at time $t_{i}$, whose path index is denoted by $l_{i \rightarrow i}$.

(ii) $\mathscr{L}_{j \rightarrow i}$ is the set of dominant paths that are firstly detected at time $t_{j}(\forall j=1,2, \ldots, i-1)$ and still exist at time $t_{i}$, whose path index is denoted by $l_{j \rightarrow i}$.

(iii) $\mathscr{L}_{i}$ is the union set of the above two sets, which contains all the dominant paths that exist at time $t_{i}$.

By detecting and tracking dominant paths in time-varying PDP and DSD, $\mathscr{L}_{i \rightarrow i}$ and $\mathscr{L}_{j \rightarrow i}$ can be constructed and the dominant multipath channel model can be reconstructed at time $t_{i}$. The dominant multipath number at time $t_{i}$ can be expressed as

$$
N\left(t_{i}\right)=N\left(t_{i \rightarrow i}\right)+\sum_{j=1}^{i-1} N\left(t_{j \rightarrow i}\right)
$$

where $N\left(t_{i \rightarrow i}\right)$ represents the number of dominant paths being newly detected at time $t_{i}$, that is, the number of elements in $\mathscr{L}_{i \rightarrow i}$; the last term of (3) refers to the dominant 
multipath number which was detected before $t_{i}$ and still exists at time $t_{i}$. Therefore, during the MRS movement, the proposed channel model is able to describe the birth and death process of dominant MPCs. Furthermore, by modeling the temporal evolution of dominant multipath behaviors in (2) during their lifetime, the proposed model can generate the exactly time-varying HST channels. Note, when a dominant path vanishes, its lifetime ends. This assumption simplifies the channel modeling and does not significantly affect the model accuracy [48].

For time-varying HST channels, parameters in (2) are generated by random geometry factors. To statistically model the geometry factor, a WSS time window should be used in a way that the statistics of geometry factor can be characterized within each WSS time window [49-51] and thereby channel modeling becomes physically meaningful. The size of WSS time window has to be defined previously. As shown in Figure 5, dominant MPCs exist in multiple consecutive WSS windows. Therefore, the lifetime of dominant path can be characterized by the WSS window number. In the proposed channel model, we use two distinct timescales to describe each path:

(i) Large-scale: the geometry factor is indexed by the WSS window index, which indicates that the propagation environment remains changeless within a WSS time window.

(ii) Small-scale: the delay, Doppler shift, and complex attenuation of dominant path are derived at each time instant using the geometry factor.

To summarize, we statistically model the following parameters:

(i) The size of dominant multipath lifetime in terms of WSS windows

(ii) The number of dominant path being newly detected in each WSS time window

(iii) The geometry factor for the newly dominant path in each WSS time window.

The developed dominant multipath model contains LoS path, FRPs, and SRPs. In practical terms, the channel model includes FRPs, SRPs, and third- or higher-order reflected paths. However, during MRS movement the energy ratio of third- or higher-order reflected paths is generally lower than $2 \%$ as shown in Figure 6 . At each time instant the energy ratio is calculated as the sum power of certain order reflected path divided by the total power of all existent reflected paths. Thus, in the proposed channel model the third- or higher-order reflected path contributions are ignored. Note that this figure is obtained from the downsampling RT simulation with the output of multipath properties. The downsampling interval is $3.6 \mathrm{~ms}$ and the corresponding distance sampling interval is $0.5 \mathrm{~m}$ (the speed of $500 \mathrm{~km} / \mathrm{h}$ ). Considering that the distances among the RRH, physical objects, and the MRS are much larger than $0.5 \mathrm{~m}$, it is reasonable to assume that during the interval no reflected path slipped away. Moreover, due to lower power contribution of diffuse multipath components

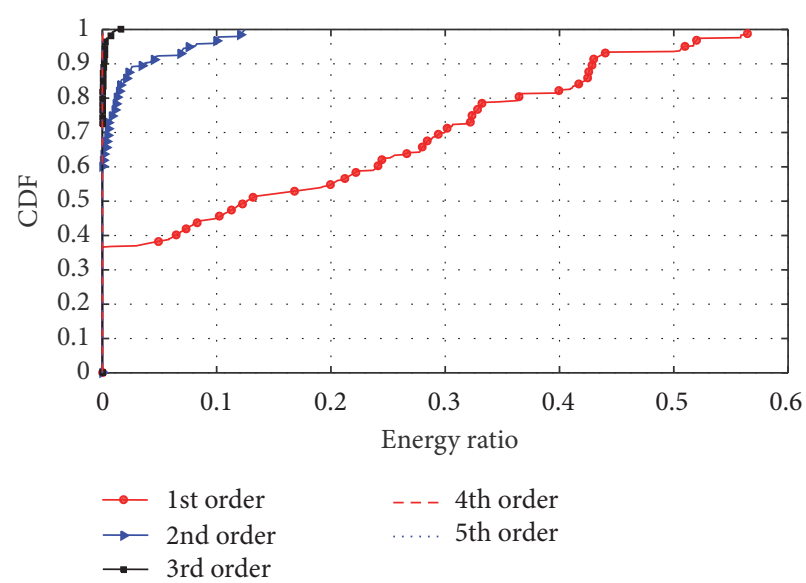

Figure 6: Energy ratio statistical distribution for different order reflected paths (order up to 5th) during the MRS movement.

(DMCs) at mmWave band DMCs are not modeled [5254]. To sum up, for the dominant multipath channel model, modeling LoS path, FRP, and SRP is sufficient.

3.2. Data Processing. Generally, in HST channel the FRPs and SRPs are reflected from the trackside walls whose normal is perpendicular to the track. As illustrated in Figure 7 the potentially geometrical characteristic is fully explored for the dominant MPCs, and the geometry factor is explicitly presented. For each dominant multipath type there is a specific right triangle, where the length of the right-angle side (which is parallel to $y$-axis) is defined as the geometry factor, that is, $\Delta y_{\mathrm{LOS}}, \Delta y_{\mathrm{FRP}}$, and $\Delta y_{\mathrm{SRP}}$. With the moving MRS $R x\left(t_{i}\right)$, the lengths of the other two sides are time-varying. It is worth noting that for LoS path the hypotenuse is equal to the path distance, while, for the FRP and SRP, the length of the hypotenuse is, according to the specular reflection law, also equal to the path distance. Hence, the multipath delays $\tau_{\mathrm{LoS}}\left(t_{i}\right), \tau_{\mathrm{FRP}}\left(t_{i}\right)$, and $\tau_{\mathrm{SRP}}\left(t_{i}\right)$ are able to be derived from the right triangles. Additionally, the respective angle between the path arrival at MRS and the MRS moving direction refers to $\theta_{\mathrm{LoS}}\left(t_{i}\right), \theta_{\mathrm{FRP}}\left(t_{i}\right)$, and $\theta_{\mathrm{SRP}}\left(t_{i}\right)$, by which the Doppler shifts for dominant MPCs can be estimated. Therefore, through the right triangles, defined mainly by the geometry factors, the delay and Doppler shift of dominant MPCs can be derived at time $t_{i}$.

Based on the analysis, the dominant multipath model can be fully characterized by the geometry factor. Moreover, by corresponding geometry factor, the temporal evolution of multipath delay and Doppler shift can be derived. Hence, we detect and track dominant MPCs by geometry factor and its geometrical relationship.

3.2.1. Dominant Multipath Detection. Before detecting the information of each dominant path (e.g., $a_{l}, \tau_{l}$, and $v_{l}$ ), the analytical expressions for the delay and Doppler shift involving the geometry factor have to be clarified. For uniformity and convenience, the subscript indicating propagation mechanism in Figure 7 is replaced with the path index $l$, where $l=\left[1, \ldots, N\left(t_{i}\right)\right]$. Note that the physical significance 


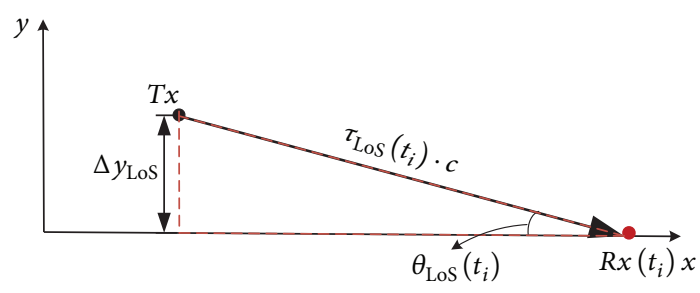

(a)

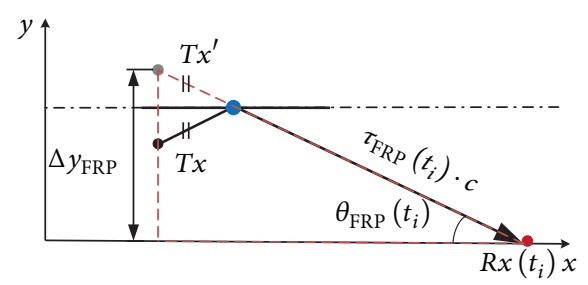

(b)

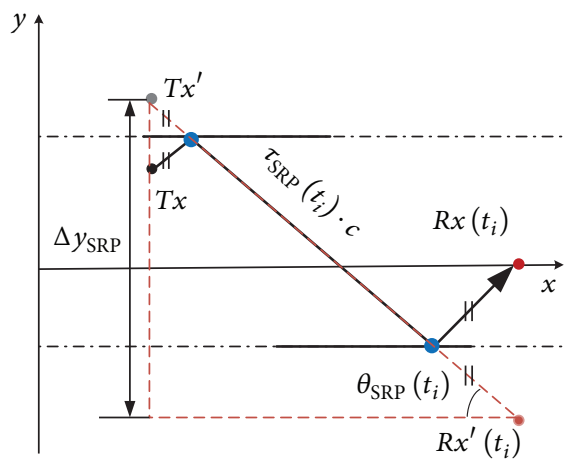

(c)

FIGURE 7: Illustration of dominant multipath geometry: (a) LoS path, (b) first-order reflected path (FRP), and (c) second-order reflected path (SRP).

for the geometry factor does not change. The position of Tx is $\left[x_{T}, y_{T}, z_{T}\right]$, and the time-varying position of MRS is $R x\left(t_{i}\right)=\left[x_{R}\left(t_{i}\right), y_{R}, z_{R}\right]$. Moreover, at $t_{i}=0$ the initial $x$ value of MRS is $x_{R}\left(t_{i}\right)=d_{c}$, where $d_{c}-x_{T}=580 \mathrm{~m}$, that is, the separation of adjacent RRHs. The length of right-angle side (which is parallel to $x$-axis) is given as

$$
\Delta x\left(t_{i}\right)=d_{c}-v \cdot t_{i}-x_{T},
$$

where $v$ denotes the MRS speed $500 \mathrm{~km} / \mathrm{h}$. As the hypotenuse length is equal to the travel distance of dominant path, the delay $\tau_{l}\left(t_{i}\right)$ is derived as

$$
\tau_{l}\left(t_{i}\right)=\frac{\sqrt{\Delta x^{2}\left(t_{i}\right)+\Delta y_{l}^{2}\left(t_{i}\right)}}{c},
$$

where $c$ refers to the speed of light and $\Delta y_{l}$ denotes the geometry factor for $l$ th path. Furthermore, the Doppler shift $v_{l}\left(t_{i}\right)$ can be given through $\theta_{l}\left(t_{i}\right)$

$$
v_{l}\left(t_{i}\right)=\frac{v}{c} \cdot \cos \left(\theta_{l}\left(t_{i}\right)\right) \cdot f_{c}
$$

where $f_{c}$ represents the carrier frequency of $93.2 \mathrm{GHz}$. Due to little height difference between $T x$ and $R x$ the elevation angle is not considered. The analytical form of $\cos \left(\theta_{l}\left(t_{i}\right)\right)$ is

$$
\cos \left(\theta_{l}\left(t_{i}\right)\right)=\frac{\Delta x\left(t_{i}\right)}{\tau_{l}\left(t_{i}\right) \cdot c} .
$$

Afterwards, by involving the geometry factor, the dominant path is detected in delay and Doppler domains. Benefitting from the dilation and erosion mathematical morphology of binary images, the trajectories in Figure 5 are refined as being

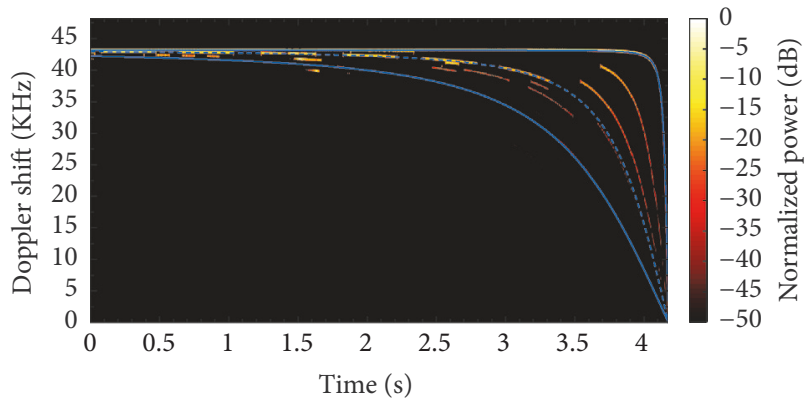

FIgURE 8: Temporal evolution of refined DSD and the Doppler trajectories for marginal geometry factors obtained (solid line). Detected geometry factor's availability is illustrated by the example trajectory (dashed line).

more clear, smooth, and coherent, as shown in Figure 8, which is beneficial for peak search. Specifically, for the image processing the time-varying DSD and PDP in Figure 5 are firstly transformed to binary image with threshold $25 \mathrm{~dB}$ [55]. The pixel with power $25 \mathrm{~dB}$ lower than the maximum power is set to 0 ; otherwise, the pixel is set to 1 . In the sth WSS time window including time instant $t_{i}$, by identifying the peaks in the refined DSD and PDP, Doppler shift and delay as well as the associated power are obtained. Each piece of peak information is stored in a peak set $\left[\widehat{\nu}_{l}\left(t_{s}\right), \widehat{\tau}_{l}\left(t_{s}\right), \widehat{p}_{l}\left(t_{s}\right)\right]$.

Subsequently, using the geometrical relation determines whether the detected peak belongs to dominant MPCs. By combining (5), (6), and (7), $\Delta x$ in (4) can be rearranged as

$$
\Delta x\left(t_{s}\right)=v_{l}\left(t_{s}\right) \cdot \tau_{l}\left(t_{s}\right) \cdot \frac{c^{2}}{v \cdot f_{c}} .
$$


To pick the dominant path from the detected sets, the detected peak set in (8) has to be incorporated and the threshold $\epsilon$ has to be used

$$
\begin{aligned}
\Delta \widehat{x}_{l}\left(t_{s}\right) & =\widehat{v}_{l}\left(t_{s}\right) \cdot \widehat{\tau}_{l}\left(t_{s}\right) \cdot \frac{c^{2}}{v \cdot f_{c}} . \\
\left|\Delta \widehat{x}_{l}\left(t_{s}\right)-\Delta x\left(t_{s}\right)\right| & \leq \epsilon
\end{aligned}
$$

where $|\cdot|$ denotes absolute value and the value $\Delta x\left(t_{s}\right)$ is obtained from (4), which is according to time-varying position of the MRS, the truth value. If the inequality is satisfied, the peak set is stored in $\mathscr{L}_{s}$ for dominant multipath modeling; otherwise, the peak set is discarded. In this analysis, we selected $\epsilon=0.9 \mathrm{~m}$ and the choices have to be done on a rather empirical basis. Afterwards, the geometry factor can be calculated as

$$
\Delta \hat{y}_{l}\left(t_{s}\right)=\Delta x\left(t_{s}\right) \cdot \sqrt{\frac{v^{2} \cdot f_{c}^{2}}{\widehat{v}_{l}\left(t_{s}\right) \cdot c^{2}}-1 .}
$$

After the detecting, for the $l$ th path within $s$ th WSS time window, there is a set $\left[\Delta \widehat{y}_{l}\left(t_{s}\right), \widehat{v}_{l}\left(t_{s}\right), \widehat{\tau}_{l}\left(t_{s}\right), \widehat{p}_{l}\left(t_{s}\right)\right]$ stored in extended set $\mathscr{L}_{s}$. For each WSS time window, the detection process will be repeated. According to the detecting, the marginal values of geometry factors are obtained, that is, the minimum and maximum values. Figure 8 shows two Doppler trajectories for marginal values (derived by (6)) and the area confined by the two which includes all the Doppler trajectories of dominant MPCs. Moreover, the example trajectory (dash line) in Figure 8 is generated by the geometry factor observed in multiple WSS time windows. Its trajectory is discontinuous with birth and death process several times, which indicates the interruptedly distributed trackside walls. Furthermore, due to applying mathematical morphology, the temporal evolution of DSD in Figure 8 is clearer and with less thin protrusions compared to the DSD in Figure 5(a).

3.2.2. Dominant Multipath Tracking. To capture the dominant multipath evolution within individual lifetimes, the tracking based on the geometry factor is performed. In two successive WSS windows, if the same geometry factor appears, its associated paths are considered to be the same path. This can be used to track the dominant path and estimate its lifetime.

For two arbitrary dominant paths $l_{u}$ and $l_{v}$, from the two successive WSS time windows, the sets of the two dominant paths are given as

$$
\begin{aligned}
& l_{u} \in \mathscr{L}_{s}:\left[\Delta \widehat{y}_{u}\left(t_{s}\right), \widehat{v}_{u}\left(t_{s}\right), \widehat{\tau}_{u}\left(t_{s}\right), \widehat{p}_{u}\left(t_{s}\right)\right] \\
& l_{w} \\
& \in \mathscr{L}_{s+1}:\left[\Delta \hat{y}_{w}\left(t_{s+1}\right), \widehat{v}_{w}\left(t_{s+1}\right), \widehat{\tau}_{w}\left(t_{s+1}\right), \widehat{p}_{w}\left(t_{s+1}\right)\right] .
\end{aligned}
$$

To enhance the tracking, the aforementioned threshold $\epsilon$ is adopted. The tracking algorithm is described as follows.

Step 1. Calculate the absolute differences between any geometry factors from $\mathscr{L}_{s}$ and $\mathscr{L}_{s+1}$ and obtain the matrix $D$ with dimension $N\left(t_{s}\right) \times N\left(t_{s+1}\right)$.

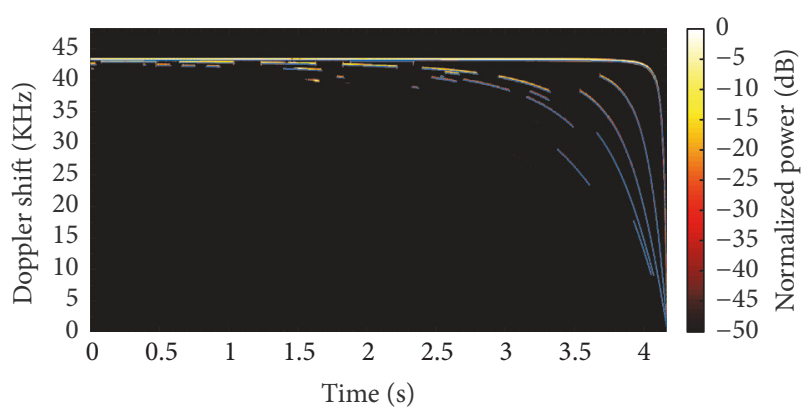

FIgURE 9: Dominant path tracking in Doppler domain.

Step 2. If the condition

$$
\begin{aligned}
D_{u, w} & \leq \epsilon \\
u & =\arg \min _{u}\left(D_{u \in N\left(t_{s}\right), w}\right) \\
w & =\arg \min _{w}\left(D_{u, w \in N\left(t_{s+1}\right)}\right)
\end{aligned}
$$

is satisfied, the $w$ th MPC at $t_{s+1}$ is considered as evolving from the $u$ th MPC at $t_{s}$. For matching, a unique path ID is assigned to them. Examine all other paths in the two successive WSS windows and match the paths evolving over time. Afterwards, if the path at $t_{s+1}$ has no ID, a new path ID is also assigned for the newly observed path.

Step 3. Repeat Steps 1 and 2 for $\mathscr{L}_{s+1}$ and $\mathscr{L}_{s+2}$, and inherit the path ID for the path evolving over multiple WSS time windows according to (12). Likewise, if new path has been observed, the new path ID is given.

Step 4. Repeat the steps for the WSS time windows after $t_{s+2}$, and coherently match in every two consecutive indices of WSS windows. Assign path IDs for all dominant paths.

Step 5. Average the geometry factors collected from the same path ID and assign the mean value as the unique geometry factor to the path ID.

Step 6. Cope with small temporal "gaps," where the information of the dominant path is missed in one or a few (smaller than $N_{\text {gap }}$ ) consecutive WSS windows. The set of the missed dominant paths can be covered through the geometry factor in adjacent WSS time windows, that is, involving the geometry factor in (5) and (6) to calculate the delay and Doppler shifts and then deriving the power by the power model presented in the next section.

The tracking algorithm starts from $s=1$ and the dominant paths at initial time are assigned to unique path IDs. In our analysis $N_{\text {gap }}=5$ is selected. By grouping dominant paths with the path ID, we obtain the lifetime and the temporal evolution for each dominant path. Figure 9 shows the outcome of the tracking algorithm in Doppler domain for the mmWave HST channel. The tracking trajectories calculated by the geometry factors (within their lifetimes) are in agreement with the time-varying DSD from RT simulation. 
TABLE 2: Conditional probabilities for newly emerging dominant multipath number.

\begin{tabular}{llccccc}
\hline & & \multicolumn{3}{c}{$N\left(t_{s_{2}-1}\right)$} & & \\
& & 1 & 2 & 3 & 5 & 5 \\
\hline \multirow{2}{*}{$N\left(t_{s_{2} \rightarrow s_{2}}\right)$} & 0 & 0.9975 & 0.9979 & 0.9990 & 0.9996 & 0.0004 \\
\hline
\end{tabular}

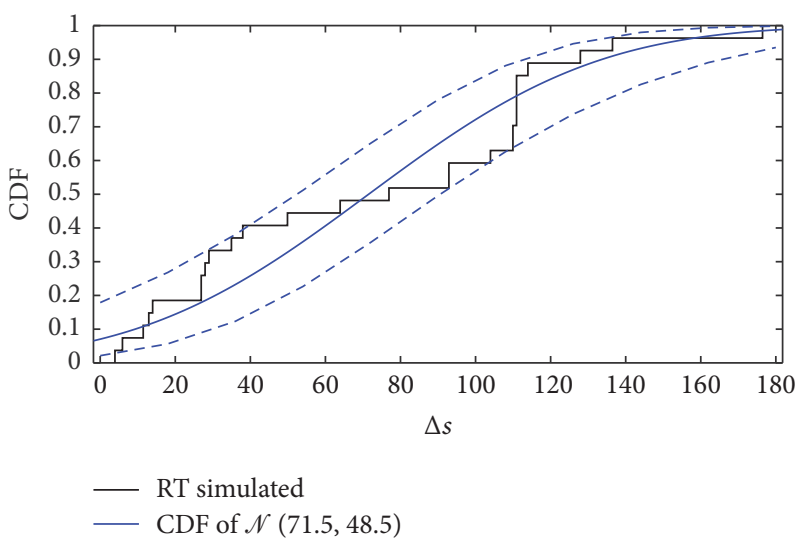

FIGURE 10: Empirical CDF of $\Delta s$ and its fitted curve with analytical expression. For the fit, two bounds at $95 \%$ confidence level are constructed (dash lines).

The good match validates the geometry-based detecting and tracking algorithm.

\section{Model Parameters}

The channel parameters are first estimated within each WSS time window $\Delta W$ and then statistically modeled.

4.1. Dominant Multipath Lifetime. If the dominant path is first observed within the $s_{1}$ th WSS window and is last observed within the $s_{2}$ th WSS window $\left(s_{1}<s_{2}\right)$, its lifetime is expressed as

$$
T=\Delta W \cdot \Delta s=\Delta W \cdot\left(s_{2}-s_{1}\right),
$$

where the index difference $\Delta s$ is used to indirectly model lifetime $T$. Note that the LoS path is not included in $\Delta s$ modeling due to its existing throughout. Figure 10 shows the empirical CDF of $\Delta s$ and it is well fitted by the truncated Gaussian distribution $\mathscr{N}(71.5,48.5)$, where 71.5 and 48.5 represent the mean value and stand deviation, respectively. In Figure $10 \Delta s$ ranges from 6 to 180 and thus the truncated Gaussian distribution is bounded within $[6,180]$. The fit is implemented by a nonlinear LS regression. To evaluate the fit, the Kolmogorov-Smirnov (KS) test at 95\% confidence level is used and it fails to reject the null hypothesis, which validates the fit. In addition, the two blue dash lines shown in Figure 10 are the confidence bounds which are constructed by the given confidence level. Obviously, the empirical CDF of $\Delta s$ is within the area limited by the two boundaries.

4.2. Newly Dominant Multipath Number. Recall (3); at time $t_{s_{2}}$ the totally dominant multipath number $N\left(t_{s_{2}}\right)$ is determined by the number of new dominant paths $N\left(t_{s_{2} \rightarrow s_{2}}\right)$ and

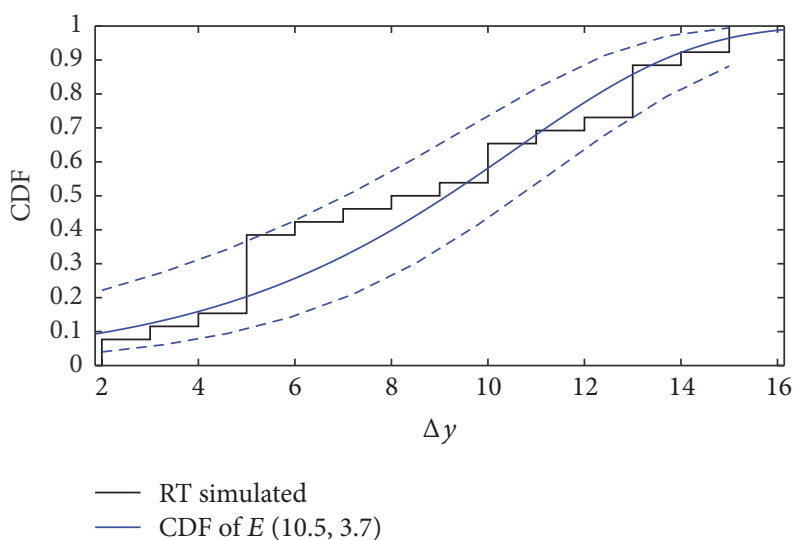

FIgURE 11: Empirical CDF of $\Delta y$ for newly dominant path and its fitted curve derived by Extreme Value distribution.

the number of old dominant paths. The old dominant paths have been observed before time $t_{s_{2}}$ and their disappearance is determined by their lifetime. Hence, only the number of new dominant paths should be modeled within each WSS time window.

For accurate modeling, the number of newly emerging dominant paths within the $s_{2}$ th time window is estimated based on dominant multipath number within the $s_{2}-$ 1)th time window. In detail, according to the conditional probabilities $P\left(N\left(t_{s_{2} \rightarrow s_{2}}\right) \mid N\left(t_{s_{2}-1}\right)\right)$ the number of newly emerging dominant paths $N\left(t_{s_{2} \rightarrow s_{2}}\right)$ can be given. In Table 2 the conditional probabilities are provided. It is evident form the table that one dominant path can be newly observed within each time window at most. Moreover, the probability of generating new path decreases when the dominant multipath number at previous time window is increased.

4.3. Newly Dominant Multipath Geometry Factor. The geometry factor is fixed during dominant multipath lifetime, and, through it, the temporal evolution of delay and Doppler shifts can be calculated by (5) and (6). Hence, when a dominant path is newly observed in a WSS time window, the geometry factor should be generated for it. It is found that the PDF of the geometry factor for the newly emerging dominant path can be modeled by Extreme Value distribution:

$$
E(x ; \mu, \sigma)=\sigma^{-1} \exp \left(\frac{x-\mu}{\sigma}\right) \exp \left(-\exp \left(\frac{x-\mu}{\sigma}\right)\right),
$$

where $E(\cdot)$ denotes the PDF function of Extreme Value distribution and location parameter $\mu$ and scale parameter $\sigma$ are model parameters that can be estimated by nonlinear LS regression method. Figure 11 shows the empirical CDF of geometry factor for newly dominant path, that is, $\Delta y$, 


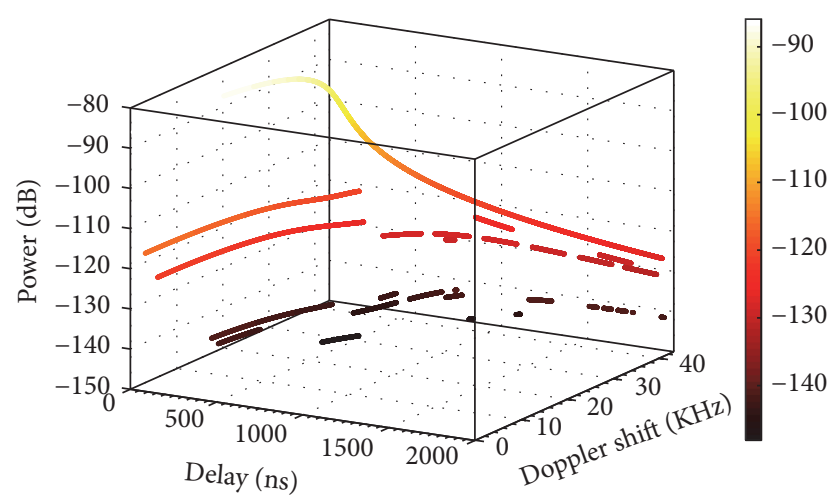

FIGURE 12: 3D scatterplot of power in $\mathrm{dB}$ versus delay and Doppler shift using the data extracted from RT simulated results.

and the LS fit for it, where the good match indicates the reasonable fit. Parameters $\mu$ and $\sigma$ are estimated as 10.5 and 3.7, respectively. Moreover, the upper and lower confidence bounds (dash lines) obtained by 95\% confidence level are presented for comparison. Furthermore, the KS test at the confidence level to evaluate the fit is adopted and the null hypothesis is accepted, which validates the fitted Extreme Value distribution for $\Delta y$.

4.4. Dominant Multipath Complex Attenuation. Recalling the parameters in (2), the number of dominant MPCs, delay, and Doppler shift have been modeled above while the complex attenuation of dominant MPCs is investigated here. Note that the parameters, that is, delay, Doppler shift, phase, and complex attenuation, are modeled using the small time-scale, that is, $t_{i}$. Figure 12 shows the $3 \mathrm{D}$ scatterplot of delay, Doppler shift, and power of dominant MPCs, which are obtained from RT simulation. Obviously, the dominant multipath path power is dependent on delay as well as Doppler shift. Generally, for the attenuation of MPCs the travel distance and angle information are the major factors. The travel distance can be obtained by the geometrical relationship, which is characterized by the delay obtained from (5). The angle information is included in Doppler shift that is derived from (6). Specifically, when the carrier frequency is given, the Doppler shift is mainly determined by the angle information. Hence, in this paper the complex attenuation is molded with combination of delay and Doppler shift.

To have a good fit to the RT simulated results, the amplitude $\left|a_{l}\left(t_{i}\right)\right|$ is modeled in decibel scale and can be divided into two parts, that is, delay-dependent part and Doppler-dependent part. Particularly, amplitude of LoS path only contains delay-dependent part

$$
20 \log 10\left(\left|a_{l}\left(t_{i}\right)\right|\right)=-20 \log 10\left(4 \pi f_{c} \cdot \tau_{l}\left(t_{i}\right)\right) .
$$

The amplitude model for the other MPCs is expressed as (16), where $\Delta y_{\mathrm{bp}}$ denotes breakpoint for the geometry factor and the parameters $p_{1}, p_{2}, p_{3}$, and $p_{4}$ for modeling Dopplerdependent part are fitted using nonlinear LS regression. Note that the Doppler shift in (16) is in decibel scale. After removing the delay-dependent part from the raw data, the power of Doppler-dependent part obviously presents piecewise behavior, shown in Figure 13, which is the reason for applying piecewise fitting. We postulate that this behavior is due to the different orders of reflection as the higher order with higher path loss. Through the geometrical analysis, the two RT simulated curves comprised of black points can be separated well from each other by the geometry factor. It is reasonable that the higher order reflected paths shall be separated with larger geometry factors, as illustrated in Figure 7. Hence, when $y<\Delta y_{\mathrm{bp}}$ indicates the FRPs, the other in (16) is for SRPs. As shown in Figure 13, the piecewise model using exponential functions offers a reasonable fit. Moreover, we note that the fitting curves for Doppler-dependent power have the same shape but different "positions." It can be considered as the exponential function with same shape parameters, that is, $p_{1}, p_{2}$, moving to different "positions." The "positions" are determined by $p_{3}$ (along $x$-axis) and $p_{4}$ (along $y$-axis). In (16), the $y$-value of "positions" for SRPs is the double of the FRPs. The fitting result gives $p_{1}=1.2$, $p_{2}=3.0, p_{3}=45.2$, and $p_{4}=-16.0$ and the breakpoint $\Delta y_{\mathrm{bp}}$ is set as $65 \mathrm{~m}$ in HST scenario.

$$
20 \log _{10}\left(\left|a_{l}\left(t_{i}\right)\right|\right)= \begin{cases}-20 \log _{10}\left(4 \pi f_{c} \cdot \tau_{l}\left(t_{i}\right)\right)+p_{1} \cdot \exp \left(p_{2} \cdot\left(v_{l}\left(t_{i}\right)-p_{3}\right)\right)+p_{4}, & \Delta y<\Delta y_{\mathrm{bp}} \\ -20 \log _{10}\left(4 \pi f_{c} \cdot \tau_{l}\left(t_{i}\right)\right)+p_{1} \cdot \exp \left(p_{2} \cdot\left(v_{l}\left(t_{i}\right)-p_{3}\right)\right)+2 p_{4}, & \Delta y \geq \Delta y_{\mathrm{bp}}\end{cases}
$$

The phase for dominant paths is expressed as

$$
\varphi_{l}\left(t_{i}\right)=-2 \pi f_{c} \tau_{l}\left(t_{i}\right) .
$$

To sum up, the complex attenuations of dominant paths can be calculated as $a_{l}\left(t_{i}\right)=\left|a_{l}\left(t_{i}\right)\right| \cdot \exp \left(j \varphi_{l}\left(t_{i}\right)\right)$.

\section{Model Implementation and Validation}

5.1. Implementation. The implementation steps of the proposed model are summarized as follows. Note that the LoS path throughout exists and thus its generation is deterministic; that is, its lifetime is equal to the duration of MRS moving; geometry factor is equal to $\Delta y_{\mathrm{LoS}}$ (see Figure 7).

(1) Initialization: set the initial dominant multipath number (range from 1 to 5 ) and the total number of modeled WSS time windows. The lifetime and geometry factor of each dominant path (except LoS path) are initialized by the results generated in Sections 4.1 and 4.3 , respectively. Then, by the geometry factor, using (5) and (6), the dominant multipath delay and 


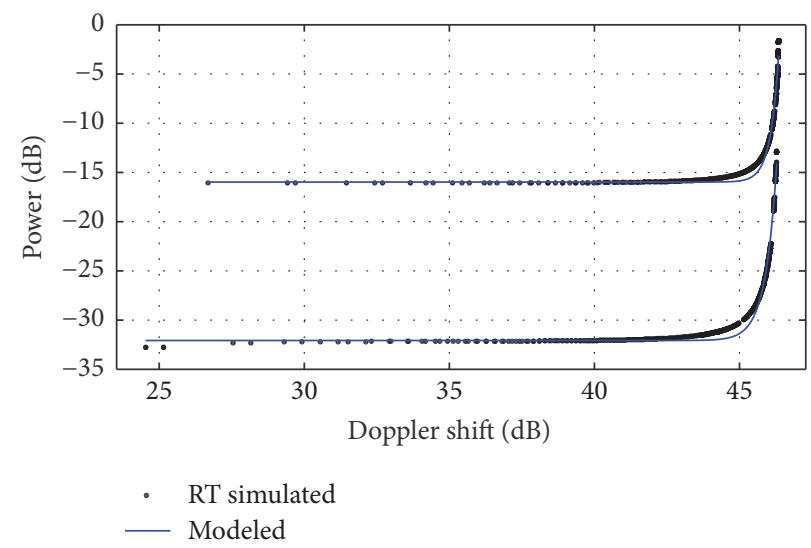

FIGURE 13: Power of Doppler-dependent part for dominant reflected path and its piecewise model obtained by nonlinear LS regression method.

Doppler shift are calculated. The complex attenuation of each initial dominant path is derived by its delay and Doppler shift by (16) and (17).

(2) Modeling for "old" MPCs: in the second WSS window the lifetime for the old dominant multipath is first checked to determine whether to be deleted. For the remaining dominant multipath, involved with the inherited geometry factors from the previous WSS window, their instantaneous delays, Doppler shifts, and complex attenuations are derived the same way as the above step.

(3) Modeling for "new" MPCs: in the second WSS window, a new dominant path generation by the procedure in Section 4.2 is checked. If new one is generated, its lifetime and geometry factor are initialized following the initialization process and then instantaneous delay, Doppler shift, and complex attenuation are derived.

(4) Temporal evolution: by repeating steps (2) and (3), the time-varying delays, Doppler shifts, and complex attenuations in spreading function (2) are derived within the consecutive WSS time windows.

5.2. Validation. The validity of the proposed model is examined by comparing the extensive model simulation with the measurement-validated RT simulation. In order to resemble the RT simulation environment, the same system parameters are set, that is, frequency, locations of $T x$ and $R x$, MRS speed, and moving range. We modeled the spreading functions for 1160 consecutive WSS time windows. Initial dominant multipath number is according to RT simulated results set to 2. Here, within each WSS window, we drop the dominant MPCs with power $25 \mathrm{~dB}$ lower than the maximum power [55]. To evaluate the accuracy of our proposed channel model, the empirical CDFs of the Root-Mean-Square (RMS) delay and Doppler spreads of the modeled channels are compared to the RT simulated ones. The RMS delay spread can be calculated as

$$
\sigma_{\tau}\left(t_{s}\right)=\sqrt{\frac{\sum_{l=1}^{N\left(t_{s}\right)} \tau_{l}\left(t_{s}\right)^{2}\left|a_{l}\left(t_{s}\right)\right|^{2}}{\sum_{l=1}^{N\left(t_{s}\right)}\left|a_{l}\left(t_{s}\right)\right|^{2}}-\bar{\tau}\left(t_{s}\right)^{2}},
$$

where the mean value $\bar{\tau}\left(t_{s}\right)$ is calculated as

$$
\bar{\tau}\left(t_{s}\right)=\frac{\sum_{l=1}^{N\left(t_{s}\right)} \tau_{l}\left(t_{s}\right)\left|a_{l}\left(t_{s}\right)\right|^{2}}{\sum_{l=1}^{N\left(t_{s}\right)}\left|a_{l}\left(t_{s}\right)\right|^{2}} .
$$

Similarly the RMS Doppler spread $\sigma_{\nu}\left(t_{s}\right)$ can be calculated by (18) with the variable $\tau_{l}\left(t_{s}\right)$ replaced by $\nu_{l}\left(t_{s}\right)$. Figure 14 depicts the empirical CDFs of $\sigma_{\tau}$ and $\sigma_{v}$, which are from RT simulated and modeled channels in Figures 14(a) and 14(b), respectively. It is shown that the model is fairly close to the RT simulation. Note that when $\sigma_{\tau}<5$ ns some differences are found between the RT simulated and modeled delay spread, which could emerge because of the model error of the geometry factor that is difficult to accurately model real scenario. Anyway, the overall performance of the mmWave HST channel model is satisfactory.

\section{Conclusion}

In this paper, a geometry-based multipath model for mmWave HST channels has been proposed. The model is developed by the RT simulator which is validated by HST measurements at $93.2 \mathrm{GHz}$. For dominant MPCs, by modeling their birth and death processes and temporal evolutions in delay and Doppler domains, the model is able to handle the time-varying characteristic in HST channels. The PDFs of the lifetime and geometry factor are fitted to truncated Gaussian distribution and Extreme Value distribution, respectively. The number of newly dominant MPCs is modeled by the conditional probabilities. To describe the complex attenuation for each dominant MPC, a new model with combination of delay and Doppler shift is proposed. In the model, delay and Doppler shift are both derived from 


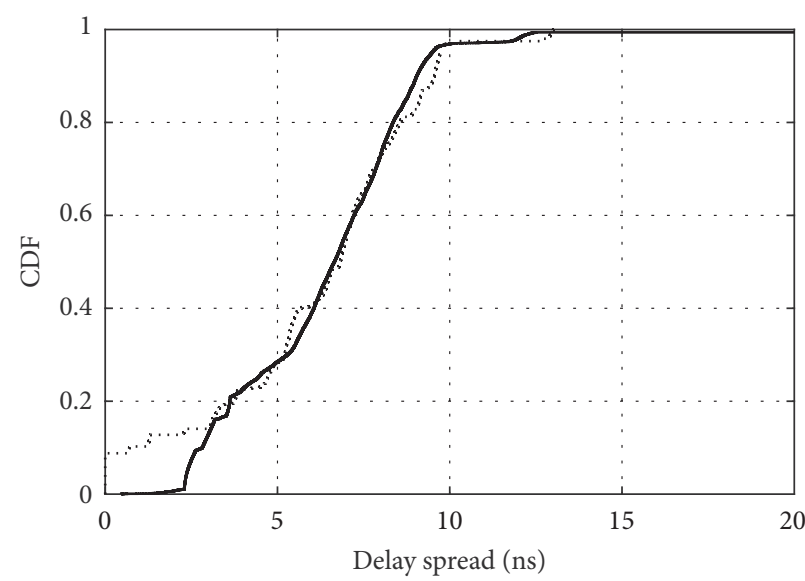

- RT simulated ..... Modeled

(a)

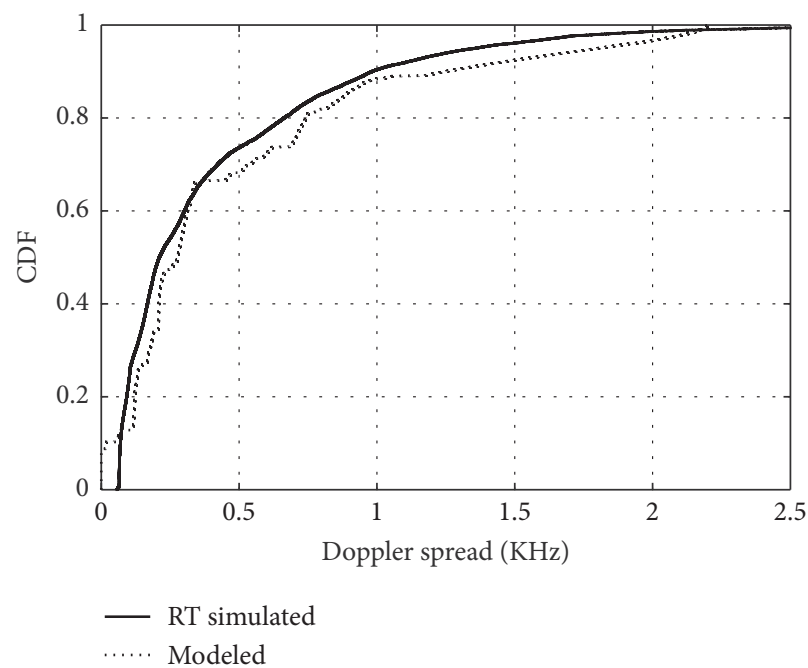

(b)

FIGURE 14: Comparisons of RMS delay spread $\sigma_{\tau}$ and Doppler spread $\sigma_{v}$ between RT simulated and modeled channels.

geometry factor. The model implementation is detailed, and it is verified by comparing the delay and Doppler spreads of the modeled channel with the RT simulated channel. The presented work shows insight into the geometry of dominant MPCs in mmWave HST scenario. Moreover, it provides an effective channel model for design and evaluation of networks in such scenario.

\section{Conflicts of Interest}

The authors declare that they have no conflicts of interest.

\section{Acknowledgments}

This work was supported in part by the National Key R\&D Program of China under Grant no. 2016YFB1200102-04, NNSF under Grants 61501021, 61771036, and U1334202, State Key Lab of Rail Traffic Control and Safety Project under Grants RCS2017ZZ004 and RCS2017ZT008, and Beijing Natural Science Foundation under Grant L161009.

\section{References}

[1] B. Ai, X. Cheng, T. Kurner et al., "Challenges toward wireless communications for high-speed railway," IEEE Transactions on Intelligent Transportation Systems, vol. 15, no. 5, pp. 2143-2158, 2014.

[2] K. Guan, G. Li, T. Kuerner et al., "On millimeter wave and THz mobile radio channel for smart rail mobility," IEEE Transactions on Vehicular Technology, vol. 66, no. 7, pp. 5658-5674, 2016.

[3] B. Ai, K. Guan, M. Rupp et al., "Future railway services-oriented mobile communications network," IEEE Communications Magazine, vol. 53, no. 10, pp. 78-85, 2015.

[4] W. Fan, I. Carton, P. Kyosti et al., "A Step Toward 5G in 2020: Low-cost OTA performance evaluation of massive MIMO base stations.", IEEE Antennas and Propagation Magazine, vol. 59, no. 1, pp. 38-47, 2017.
[5] C. X. Wang, A. Ghazal, B. Ai, Y. Liu, and P. Fan, "Channel measurements and models for high-speed train communication systems: A survey," IEEE Communications Surveys Tutorials, vol. 18, no. 2, pp. 974-987, 2016.

[6] “Updated high speed train scenario desciption," Tech. Rep. R1163867, 2016.

[7] "WF on evaluation assumptions for high speed train scenario: Macro + relay at $30 \mathrm{GHz}$," Tech. Rep. R1-165484, Etri, Korea, May 2016.

[8] X. Chen and S. Zhang, "Multiplexing efficiency for MIMO antenna-channel impairment characterisation in realistic multipath environments," IET Microwaves, Antennas \& Propagation, vol. 11, no. 4, pp. 524-528, 2017.

[9] C. Gustafson, K. Haneda, S. Wyne, and F. Tufvesson, "On mm-wave multipath clustering and channel modeling," IEEE Transactions on Antennas and Propagation, vol. 62, no. 3, pp. 1445-1455, 2014.

[10] M. K. Samimi and T. S. Rappaport, "3-D millimeter-wave statistical channel model for $5 \mathrm{G}$ wireless system design," IEEE Transactions on Microwave Theory and Techniques, vol. 64, no. 7, pp. 2207-2225, 2016.

[11] B. Ai, K. Guan, R. He et al., "On indoor millimeter wave massive mimo channels: measurement and simulation," IEEE Journal on Selected Areas in Communications, vol. 35, no. 7, pp. 1678-1690, 2017.

[12] D. He, K. Guan, B. Ai et al., "Channel modeling for Kiosk downloading communication system at $300 \mathrm{GHz}$," in Proceedings of the 2017 11th European Conference on Antennas and Propagation (EUCAP), pp. 1331-1335, Paris, France, March 2017.

[13] W. Fan, I. Carton, J. Ø. Nielsen, K. Olesen, and G. F. Pedersen, "Measured wideband characteristics of indoor channels at centimetric and millimetric bands," Eurasip Journal on Wireless Communications and Networking, vol. 2016, no. 1, article no. 58, pp. 1-13, 2016.

[14] S. Knörzer, M. A. Baldauf, T. Fügen, and W. Wiesbeck, "Channel analysis for an OFDM-MISO train communications system using different antennas," in Proceedings of the 2007 IEEE 66th Vehicular Technology Conference, VTC 2007-Fall, pp. 809-813, October 2007. 
[15] K. Guan, Z. Zhong, B. Ai, and T. Kürner, "Deterministic propagation modeling for the realistic high-speed railway environment," in Proceedings of the IEEE 77th Vehicular Technology Conference (VTC '13), pp. 1-5, Dresden, Germany, June 2013.

[16] L. Tian, X. Yin, Q. Zuo, J. Zhou, Z. Zhong, and S. X. Lu, "Channel modeling based on random propagation graphs for high speed railway scenarios," in Proceedings of the IEEE 23rd International Symposium on Personal, Indoor and Mobile Radio Communications (PIMRC '12), pp. 1746-1750, Sydney, Australia, September 2012.

[17] Y. Chang, M. Furukawa, H. Suzuki, and K. Fukawa, "Propagation analysis with ray tracing method for high speed trains environment at $60 \mathrm{GHz}$," in Proceedings of the 81st IEEE Vehicular Technology Conference (VTC Spring '15), pp. 1-5, Glasgow, Scotland, May 2015.

[18] K. Guan, X. Lin, D. He et al., "Scenario modules and raytracing simulations of millimeter wave and terahertz channels for smart rail mobility," in Proceedings of the 2017 11th European Conference on Antennas and Propagation (EUCAP), pp. 113-117, Paris, France, March 2017.

[19] M. Walter, D. Shutin, and U.-C. Fiebig, "Delay-dependent doppler probability density functions for vehicle-to-vehicle scatter channels," IEEE Transactions on Antennas and Propagation, vol. 62, no. 4, pp. 2238-2249, 2014.

[20] R. He, Z. Zhong, B. Ai, J. Ding, Y. Yang, and A. F. Molisch, "Short-term fading behavior in high-speed railway cutting scenario: Measurements, analysis, and statistical models," IEEE Transactions on Antennas and Propagation, vol. 61, no. 4, pp. 2209-2222, 2013.

[21] R. He, B. Ai, Z. Zhong, A. F. Molisch, R. Chen, and Y. Yang, "A measurement-based stochastic model for high-speed railway channels," IEEE Transactions on Intelligent Transportation Systems, vol. 16, no. 3, pp. 1120-1135, 2015.

[22] K. Guan, Z. Zhong, B. Ai, and T. Kurner, "Propagation measurements and analysis for train stations of high-speed railway at $930 \mathrm{MHz}$," IEEE Transactions on Vehicular Technology, vol. 63, no. 8, pp. 3499-3516, 2014.

[23] R. He, Z. Zhong, B. Ai, G. Wang, J. Ding, and A. F. Molisch, "Measurements and analysis of propagation channels in highspeed railway viaducts," IEEE Transactions on Wireless Communications, vol. 12, no. 2, pp. 794-805, 2013.

[24] S. Lin, L. Kong, L. He et al., "Finite-state Markov modeling for high-speed railway fading channels," IEEE Antennas and Wireless Propagation Letters, vol. 14, pp. 954-957, 2015.

[25] L. Liu, C. Tao, J. Qiu et al., "Position-based modeling for wireless channel on high-speed railway under a viaduct at $2.35 \mathrm{GHz}$," IEEE Journal on Selected Areas in Communications, vol. 30, no. 4, pp. 834-845, 2012.

[26] Y. Zhang, Z. He, W. Zhang, L. Xiao, and S. Zhou, "Measurementbased delay and doppler characterizations for high-speed railway hilly scenario," International Journal of Antennas and Propagation, vol. 2014, Article ID 875345, 8 pages, 2014.

[27] K. Guan, G. Li, D. He et al., "Spatial consistency of dominant components between ray-tracing and stochastic modeling in 3GPP high-speed train scenarios," in Proceedings of the 2017 11th European Conference on Antennas and Propagation (EUCAP), pp. 3182-3186, Paris, France, March 2017.

[28] P. Bello, "Characterization of randomly time-variant linear channels," IEEE Transactions on Communications, vol. 11, no. 4, pp. 360-393, 1963.

[29] D. Cichon, T. Zwick, and W. Wiesbeck, "Ray optical modeling of wireless communications in high-speed railway tunnels," in
Proceedings of the Vehicular Technology Conference - VTC, pp. 546-550, Atlanta, GA, USA.

[30] L. Bernado, T. Zemen, F. Tufvesson, A. F. Molisch, and C. F. Mecklenbrauker, "Delay and doppler spreads of nonstationary vehicular channels for safety-relevant scenarios," IEEE Transactions on Vehicular Technology, vol. 63, no. 1, pp. 82-93, 2014.

[31] A. F. Molisch, "A generic model for MIMO wireless propagation channels in macro- and microcells," IEEE Transactions on Signal Processing, vol. 52, no. 1, pp. 61-71, 2004.

[32] J. Meinilä, P. Kyösti, T. Jämsä, and L. Hentilä, "WINNER II channel models," Tech. Rep. WINNER II D1.1.2 IST-4-027756, 2008.

[33] T. A. Thomas, F. W. Vook, E. Visotsky, E. Mellios, G. S. Hilton, and A. R. Nix, "3D extension of the 3GPP/ITU channel model," in Proceedings of the 2013 IEEE 77th Vehicular Technology Conference, VTC Spring 2013, Dresden, Germany, June 2013.

[34] B. Chen, Z. Zhong, B. Ai, and D. G. Michelson, "A geometrybased stochastic channel model for high-speed railway cutting scenarios," IEEE Antennas and Wireless Propagation Letters, vol. 14, pp. 851-854, 2015.

[35] A. Ghazal, C.-X. Wang, B. Ai, D. Yuan, and H. Haas, "A nonstationary wideband MIMO channel model for highmobility intelligent transportation systems," IEEE Transactions on Intelligent Transportation Systems, vol. 16, no. 2, pp. 885-897, 2015.

[36] B. Ai, R. He, Z. Zhong et al., "Radio wave propagation scene partitioning for high-speed rails," in Proceedings of the Oct, vol. 2012, pp. 1072-1075, 2012.

[37] X. Yin, X. Cai, X. Cheng, J. Chen, and M. Tian, "Empirical geometry-based random-cluster model for high-speed-train channels in UMTS networks," IEEE Transactions on Intelligent Transportation Systems, vol. 16, no. 5, pp. 2850-2861, 2015.

[38] J. Karedal, F. Tufvesson, N. Czink et al., "A geometry-based stochastic MIMO model for vehicle-to-vehicle communications," IEEE Transactions on Wireless Communications, vol. 8, no. 7, pp. 3646-3657, 2009.

[39] T. Kawanishi, A. Kanno, H. Ogawa et al., "Proposal of a new working document of a draft new apt report on millimeter-wave band railway radiocommunication systems between train and trackside, and its work plan," Tech. Rep. AWG-20/INP-43, National Institute of Information and Communication technology, Bangkok, Japan, 2016.

[40] "Coexistence between railway radiocommunication system between train and trackside operating in the frequency bands 92-94 GHz, 94.1-100 GHz and 102-109.5 GHz, and active and passive services," Tech. Rep. Annex 30, National Institute of Information and Communication technology, Geneva, Switzerland, 2017.

[41] K. Nakamura, K. Kawasaki, K. Takeuchi, N. Yonemoto, A. Kohmura, and S. Futatsumori, "Radio propagation characteristics of the $90 \mathrm{GHz}$ millimeter-wave in railway environment," IEICE technical report, vol. 113, no. 333, pp. 33-38, 2013.

[42] J. Zhang, Y. Zhang, Y. Yu, R. Xu, Q. Zheng, and P. Zhang, “3D MIMO: how much does it meet our expectations observed from channel measurements?" IEEE Journal on Selected Areas in Communications, vol. 35, no. 8, pp. 1887-1903, 2017.

[43] "Discussion on time domain frame structure for high speed train scenario with relay for NR," Tech. Rep. R1-1609609, Etri, Korea, 2016.

[44] "Discussion on the subcarrier spacing selection for NR," Tech. Rep. R1-1609608, Etri, Korea, 2016. 
[45] R. He, O. Renaudin, V.-M. Kolmonen et al., "A dynamic wideband directional channel model for vehicle-to-vehicle communications," IEEE Transactions on Industrial Electronics, vol. 62, no. 12, pp. 7870-7882, 2015.

[46] G. Matz, "On non-WSSUS wireless fading channels," IEEE Transactions on Wireless Communications, vol. 4, no. 5, pp. 2465-2478, 2005.

[47] L. Bernardó, A. Roma, A. Paier et al., "In-tunnel vehicular radio channel characterization," in Proceedings of the IEEE 73rd Vehicular Technology Conference (VTC '11), Budapest, Hungary, May 2011.

[48] N. Czink, C. Mecklenbräuker, and G. Del Galdo, "A novel automatic cluster tracking algorithm," in Proceedings of the 2006 IEEE 17th International Symposium on Personal, Indoor and Mobile Radio Communications, PIMRC, Helsinki, Finland, September 2006.

[49] C.-I. Chen and Y.-C. Chen, "Comparative study of harmonic and interharmonic estimation methods for stationary and timevarying signals," IEEE Transactions on Industrial Electronics, vol. 61, no. 1, pp. 397-404, 2014.

[50] S. K. Jain, S. N. Singh, and J. G. Singh, "An adaptive timeefficient technique for harmonic estimation of nonstationary signals," IEEE Transactions on Industrial Electronics, vol. 60, no. 8, pp. 3295-3303, 2013.

[51] J. Zhang, C. Pan, F. Pei, G. Liu, and X. Cheng, "Threedimensional fading channel models: a survey of elevation angle research," IEEE Communications Magazine, vol. 52, no. 6, pp. 218-226, 2014.

[52] S. Salous, V. Degli Esposti, F. Fuschini et al., "MillimeterWave Propagation: Characterization and modeling toward fifthgeneration systems. [Wireless Corner]," IEEE Antennas and Propagation Magazine, vol. 58, no. 6, pp. 115-127, 2016.

[53] D. Dupleich, F. Fuschini, R. Mueller et al., "Directional characterization of the $60 \mathrm{GHz}$ indoor-office channel," in Proceedings of the 31st General Assembly and Scientific Symposium of the International Union of Radio Science, URSI GASS 2014, Beijing, China, August 2014.

[54] K. Haneda, J. Järveläinen, A. Karttunen, M. Kyrö, and J. Putkonen, "A statistical spatio-temporal radio channel model for large indoor environments at 60 and $70 \mathrm{GHz}$," Institute of Electrical and Electronics Engineers. Transactions on Antennas and Propagation, vol. 63, no. 6, pp. 2694-2704, 2015.

[55] D. W. Matolak, "Channel modeling for vehicle-to-vehicle communications," IEEE Communications Magazine, vol. 46, no. 5, pp. 76-83, 2008. 


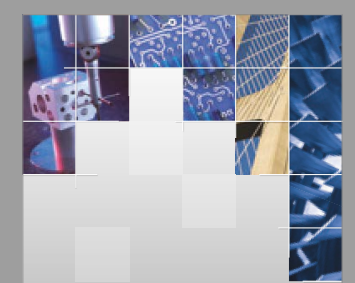

\section{Enfincering}
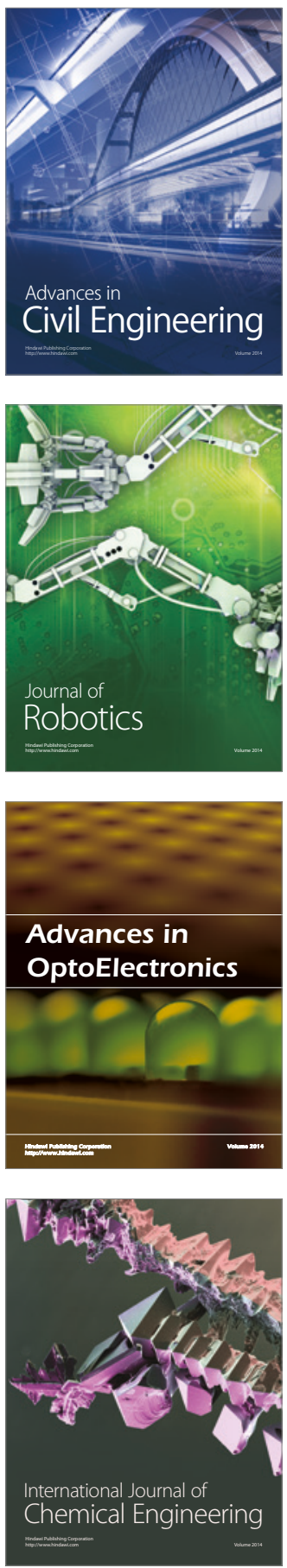

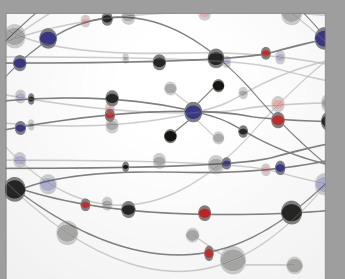

The Scientific World Journal

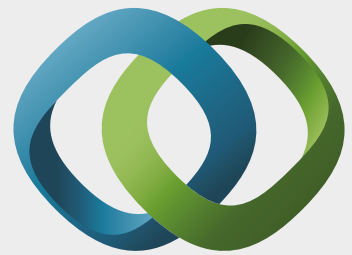

\section{Hindawi}

Submit your manuscripts at

https://www.hindawi.com
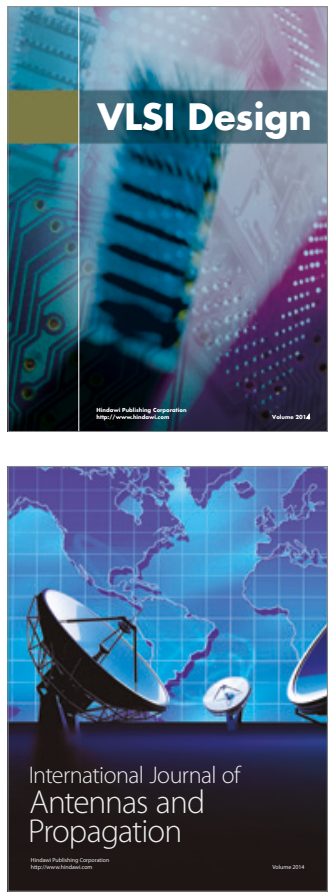

\section{Rotating}

Machinery
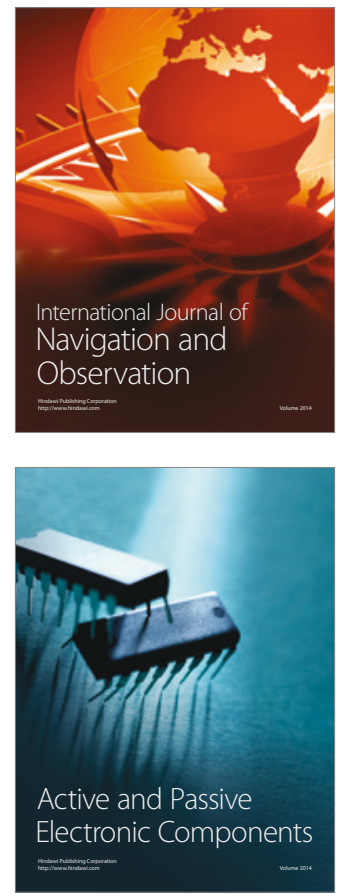
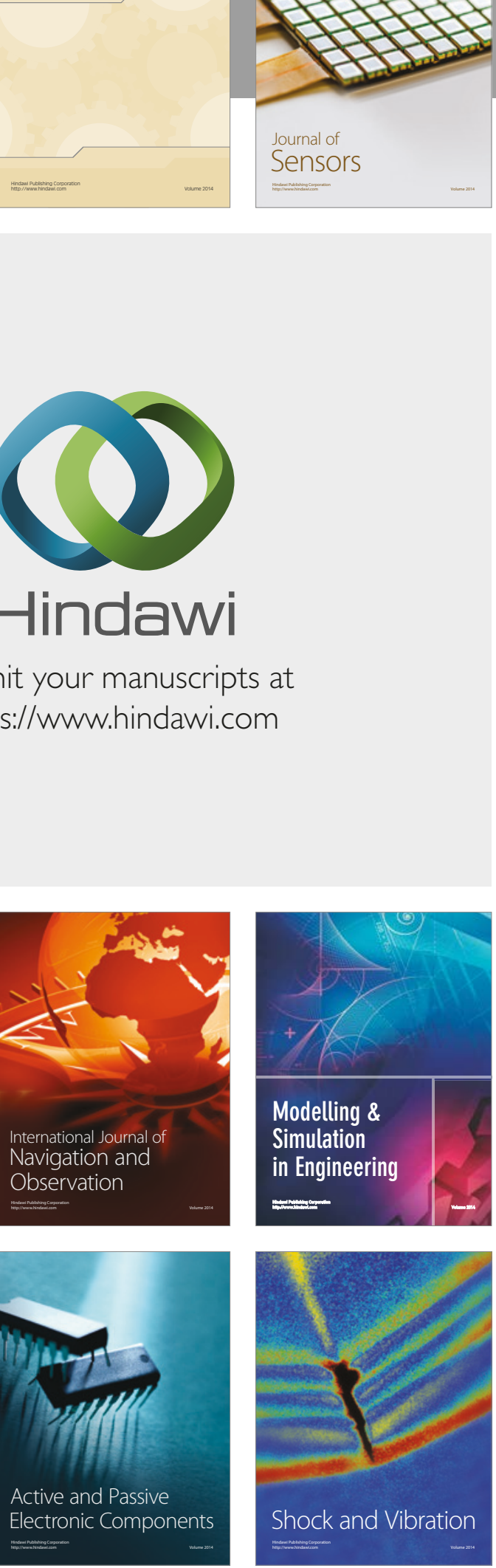
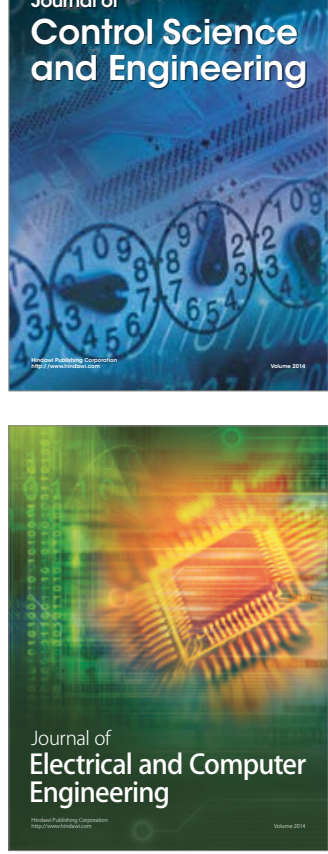

Distributed

Journal of

Control Science

and Engineering
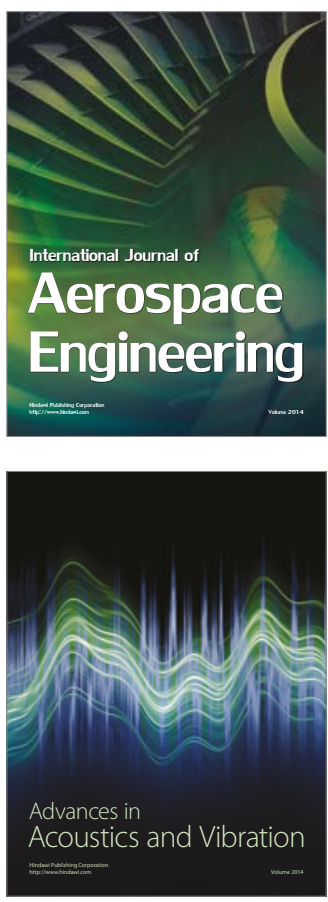

Sensor Networks 